\title{
Desenvolvimento de robôs diferenciais futebolistas: uma aplicação de campos vetoriais no planejamento de caminho
}

\author{
Paulo Henrique dos Santos * Alexandre Magno de S Thiago Filho* \\ Elias José de Rezende Freitas ** \\ * Universidade Federal de Ouro Preto (UFOP), MG. \\ ** Instituto Federal de Minas Gerais - Campus Avançado Itabirito - MG
}

\begin{abstract}
Mobile robots can perform different types of tasks, including to play soccer. This work presents the development of differential robots to compete in the category IEEE Very Small Size Soccer (VSSS). In addition to presenting a path planning based on vector fields, combining an attractive and a repulsive field. The results and comparisons made with the real robots point out that the adopted technique allows a gain in the efficiency of the robot during the match, since it also reaches the ball with the orientation defined by the general strategy of game, for example, with the orientation aimed at the goal, allows the robot to avoid from possible obstacles in its path, reducing the time spent in collisions and a possible strategy of marking the opponent.

Resumo: Robôs móveis podem realizar diferentes tipos de tarefas, dentre elas a de jogar futebol. Neste trabalho é apresentado de maneira geral o desenvolvimento de robôs diferenciais para competir na categoria IEEE Very Small Size Soccer (VSSS). Adicionalmente, apresenta-se um planejamento de caminho baseado em campos vetoriais, sendo esse uma combinação de um campo atrativo e outro repulsivo. Os resultados e comparações realizadas com os robôs reais apontam que a técnica adotada permite um ganho na eficiência do robô durante o jogo, já que o mesmo além de chegar à bola com a orientação definida pela estratégia geral de jogo, por exemplo, com a orientação apontada para o gol, permite que o robô desvie dos possíveis obstáculos em seu caminho, evitando o tempo gasto nas colisões e de uma possível estratégia de marcação do adversário.
\end{abstract}

Keywords: Mobile Robots; Path Planning; Robot Soccer; Vector Field.

Palavras-chaves: Robôs Móveis; Planejamento de Caminho; Futebol de Robôs; Campo vetorial.

\section{INTRODUÇÃO}

A robótica móvel é uma área que trata dos robôs que possuem a capacidade de se locomover em um ambiente, seja ele terrestre, aéreo ou aquático. A autonomia com que os robôs se locomovem e interagem com o ser humano permite classificá-los em: (i) tele-operados: totalmente dependente do ser humano; (ii) semiautônomos: parcialmente dependente do ser humano, sendo que as tarefas, principalmente, relacionadas a segurança da navegação são de responsabilidade do robô; (iii) autônomos: totalmente independente do ser humano, todas as tarefas executadas não necessitam da intervenção humana (Freitas et al., 2017).

Um exemplo de sistema robótico complexo que envolve a interação de multi-robôs autônomos é o futebol de robôs. Esse jogo, semelhante ao humano, mas com algumas regras modificadas, é vencido pela equipe de robôs que faz o maior número de gols no adversário. Na Figura 1 é apresentada a visualização de uma partida da categoria IEEE Very Small Size Soccer (VSSS), utilizando a plataforma VSS-Viewer no modo simulação. Sendo possível observar que nessa categoria o jogo é composto por duas equipes de três robôs, sendo cada equipe identificada pela cor amarela ou azul e cada robô por uma cor específica.

Para tal, cada robô jogador deve ser capaz de navegar pelo campo de forma conjunta com os demais, sendo necessário,

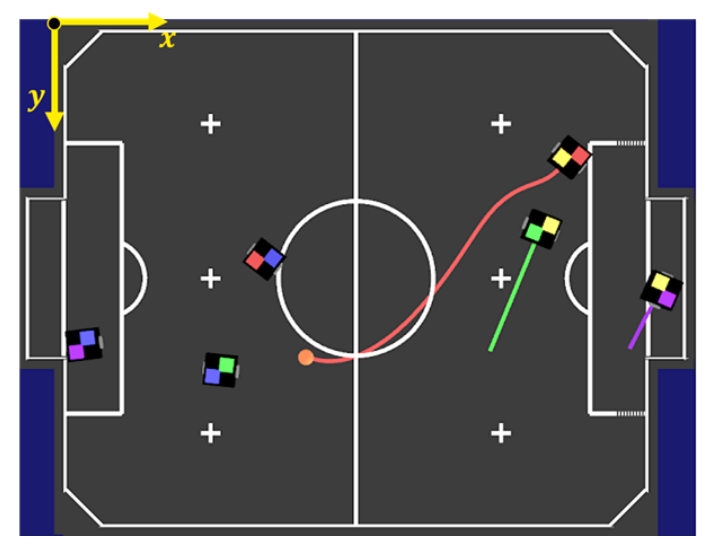

Figura 1. Visualização de uma partida da categoria IEEE Very Small Size Soccer, utilizando a plataforma VSS-Viewer no modo simulação. As linhas coloridas indicam o caminho a ser seguido pelo robô no instante da foto.

assim, calcular a cada instante um caminho a ser seguido. Esse tipo de problema é conhecido como planejamento de caminho. Para resolvê-lo existem diferentes soluções, como aquelas que utilizam algoritmos probabilísticos, por exemplo, baseadas no RRT*, e técnicas reativas, como aquelas baseadas em campos potenciais. Porém, a solução mais comum no caso 
de robôs jogadores de futebol da categoria VSSS são aquelas que utilizam campos vetoriais para guiar o robô, sendo que a integração desse campo a partir de um ponto inicial fornece o caminho planejado.

O trabalho de Li et al. (2003) é um exemplo desse tipo de planejamento de caminho aplicado aos robôs jogadores de futebol da categoria VSS. Nele um campo vetorial unitário é gerado por meio de lógica Fuzzy, permitindo que o robô além de alcançar um determinado alvo, normalmente, a bola ou o gol adversário, desvie de obstáculos em seu percurso (robôs adversários ou do próprio time). Já em (Kim et al., 2001) e (Kim et al., 2010) os autores propõem um campo vetorial construído de tal maneira a, não apenas, levar o robô ao alvo, mas alcançá-lo com uma orientação desejada. Esse aspecto é muito importante durante a partida, já que essa orientação pode ser utilizada pela estratégia de jogo, por exemplo, para permitir que o robô alcance a bola e já esteja orientado para o gol adversário. Em outros trabalhos encontram-se diferentes maneiras para a criação desses campos vetoriais, como utilizando técnicas de reinforcement learning (Park et al., 2001), (Vien et al., 2007) e até mesmo um algoritmo evolutivo que permite o desvio de obstáculos, tendo em conta as velocidades do robô e dos obstáculos ao seu redor (Lim et al., 2008).

Além de planejar o caminho, é necessário desenvolver um controle capaz de assegurar o seu seguimento. Tipicamente os controles mais aplicados aos robôs diferenciais são: (i) controle por modo deslizante (Kim et al., 2001) e (Junkai et al., 2016) e (ii) controle por realimentação entrada-saída (feedback linearization) (Hassan, 2014) e (He, 2005).

Em síntese, este artigo apresenta de maneira abrangente o desenvolvimento de robôs diferenciais aplicados ao futebol de robôs da categoria VSSS, incluindo o sistema de hardware e de software, a estratégia geral de jogo, o planejamento de caminho e o controle para o seu seguimento. Além de possibilitar essa visão global do assunto, pode-se citar ainda como contribuição deste artigo a adaptação/combinação de duas técnicas para o planejamento de caminhos em ambientes dinâmicos, baseados em campos vetoriais. Dessa forma, este artigo apresenta resultados promissores que confirmam que o robô é capaz de desviar dos demais robôs presentes no ambiente e ainda consiga, se for possível, chegar até a bola com uma orientação pré-definida pela estratégia geral de jogo.

As próximas seções deste artigo são organizadas como segue. A Seção 2 apresenta o sistema como um todo. A estratégia geral do jogo é descrita na Seção 3 e o planejamento de movimento dos robôs na Seção 4. Já a Seção 5 é dedicada ao controle necessário para garantir o seguimento dos caminhos fornecidos pelo planejador. Os experimentos e resultados são apresentados e discutidos na Seção 6. Por fim, a Seção 7 expõe as conclusões e aponta os trabalhos futuros.

\section{DESCRIÇÃO DO SISTEMA}

$\mathrm{Na}$ categoria de futebol de robôs VSSS duas equipes disputam com três robôs em cada time, sendo que cada um desses deve ter o formato delimitado por um cubo de no máximo $7,5 \times 7,5 \times 7,5 \mathrm{~cm}^{3}$ e devem ser controlados remotamente por um computador, sem a intervenção humana, ou seja, de forma autônoma.

Dessa forma, o desenvolvimento dos robôs futebolistas pode ser dividido em duas etapas: (i) projeto do sistema embarcado (hardware e firmware) e (ii) projeto do sistema de processamento externo do robô.

\subsection{Sistema embarcado}

O hardware dos robôs futebolistas é constituído dos seguintes elementos:

- Motores: O acionamento de cada roda do robô é realizado por um micro motor $\mathrm{CC}$ da Pololu, com caixa de redução de 51,45:1 e rotação à vazio de 590rpm@6V.

- Fonte de alimentação: A alimentação dos circuitos eletrônicos é obtida pela combinação de uma bateria lítioíon (tensão nominal de 7,4V, capacidade de $1000 \mathrm{mAh}$ e regime de descarga de 25C) e um conversor CC-CC buck, ajustado para manter uma tensão constante de $6 \mathrm{~V}$ (tensão nominal dos motores).

- Processador: O processamento do sistema embarcado é realizado por uma placa de desenvolvimento Arduino Nano, baseada no microcontrolador Atmega328P.

- Driver de potência: Como a placa de desenvolvimento escolhida não possui potência suficiente para acionar os motores, foi necessário a utilização de uma ponte-H, baseada no TB6612FNG.

- Módulo wifi de comunicação: Para garantir a comunicação entre o sistema embarcado e o computador externo é utilizado um módulo Xbee que opera dentro da faixa de frequência de 2,4 GHz. Esse módulo tem baixo consumo de energia e uma entrega confiável dos dados.

\subsection{Sistema de processamento externo}

O processamento externo é realizado por um computador Intel Core i5 Quad Core de 3.0 GHz com 8 GB de RAM. A taxa de processamento é realizada com base nas informações obtidas de uma câmera, que fornece a visão superior do campo à $100 \mathrm{~Hz}$. Todo o software desenvolvido é realizado sobre o projeto open source: VSS-SDK ${ }^{1}$. As principais tarefas do processamento externo são: (i) processamento e obtenção de informações (localização dos robôs no campo) a partir da imagem fornecida por uma câmera (visão computacional); (ii) estratégia geral do jogo e (iii) pelo planejamento do movimento de cada robô.

\section{ESTRATÉGIA GERAL}

A estratégia é responsável por definir a função de cada robô em campo: (i) atacante; (ii) zagueiro e (iii) goleiro. O atacante é o robô que estiver posicionado na região que vai do meio de campo até o gol adversário, dessa forma, a todo instante o seu objetivo é seguir a bola e conduzi-la ao gol. O zagueiro realiza uma linha defensiva atrás do meio campo, de maneira que se a bola estiver no ataque ele apenas acompanha a direção da mesma, caso a bola esteja na região defensiva ele deve segui-la e, quando estiver próximo à bola, o robô irá girar em torno do seu próprio eixo, com o intuito de afastá-la, mais rapidamente, daquela região, dificultando a chegada do adversário. $\mathrm{O}$ goleiro é o jogador mais recuado do time, posicionado dentro da área, também, forma uma linha que acompanha a direção da bola, essa linha no entanto é mais restrita, realizando assim menores movimentos, afim de não expor o gol a ataques dos adversários.

\footnotetext{
1 O projeto VSS-SDK foi criado com o intuito de auxiliar equipes iniciantes, dando uma estrutura de tudo que é necessário para participar da categoria VSSS. Nos últimos anos, a equipe Rodetas da UFOP, da qual os autores fazem parte, tem contribuído no aprimoramento desse projeto: https://github. com/VSS-SDK.
} 


\section{PLANEJAMENTO DE CAMINHO}

O planejamento de caminho é responsável por calcular o movimento necessário que o robô deve executar para uma dada tarefa. Neste trabalho duas técnicas foram combinadas e adaptadas para a composição de um campo vetorial que tem como objetivo direcionar o robô até um alvo e alcançá-lo com uma orientação desejada, além de garantir o desvio dos obstáculos que estão presentes no percurso. Dessa forma, um vetor unitário $f(p)$, definido para cada posição possível para o robô em seu ambiente, $p=[x, y]^{\prime}$, guia o robô ao objetivo estabelecido:

$$
f: \mathscr{C} \rightarrow V
$$

em que $p \in \mathscr{C}$, o espaço de trabalho do robô, no caso, definido em $\mathbb{R}^{2}$, e $V$ é o campo vetorial, formado pelo conjunto de vetores unitários. Como o vetor criado é unitário, pode-se representá-lo apenas em termo de uma função angular

$$
\phi(p): \mathscr{C} \rightarrow[-\pi, \pi]
$$

Para facilitar o entendimento e apresentar as etapas de criação desse vetor unitário, o planejamento foi dividido em três etapas, descritos nas próximas subseções: (i) criação de um campo vetorial atrativo capaz de guiar o robô para que o mesmo chegue a um determinado alvo com a orientação final desejada, definido no trabalho de Kim et al. (2001); (ii) criação de um campo vetorial destinado ao desvio dos obstáculos que existem no campo, definida em (Lim et al., 2008), mas que é uma maneira genérica de criar um campo repulsivo em torno do obstáculo e (iii) composição dos campos (i) e (ii), gerando um campo vetorial final. Essa composição é uma das contribuições deste trabalho. Pelo conhecimento dos autores, essa combinação ainda não foi testada e como será apresentado na Seção 6 foi validada com sucesso.

\subsection{Campo vetorial atrativo (CVA)}

O campo vetorial atrativo (CVA) possibilita que o robô, seguindo o vetor unitário para uma dada posição e a cada instante, alcance o alvo com uma orientação desejada. Sendo esse vetor unitário, pode-se representá-lo apenas por uma função angular $\phi_{C V A}(p)$, tal que: $\phi_{C V A}: \mathscr{C} \rightarrow[-\pi, \pi]$, definida por:

$$
\phi_{C V A}(p)=(1+n) \cdot\langle\overrightarrow{p g}-n \cdot\langle\overrightarrow{p r}
$$

em que $n$ é uma constante de balanceamento que permite alterar o raio da curva executada pelo robô ${ }^{2}$. Na Figura 2 é possível visualizar o campo vetorial atrativo criado para uma determinada situação, o caminho planejado (em vermelho), o ponto $g$ que representa o objetivo, o ponto $r$ que define a orientação de chegada ao objetivo e o ponto $p$ que é uma posição qualquer no campo.

Como percebido pela Equação 1, o cálculo do ângulo para uma dada posição $p$ depende apenas da orientação de dois vetores, sendo eles $\overrightarrow{p g}$ e $\overrightarrow{p r}$. Assim, além da constante $n$, a distância entre os pontos $g$ e $r$, também, afetam no raio da curva ${ }^{3}$. Mais detalhes em (Kim et al., 2001).

\subsection{Campo vetorial para o desvio de obstáculos (CVD)}

Uma maneira intuitiva que permite o desvio de obstáculos é a criação de um campo repulsivo próximo a eles. Inicialmente,

\footnotetext{
2 Note que notação $/ \overrightarrow{\mathbf{i j}}$ representa o ângulo do vetor que vai do ponto $i$ ao ponto $j$.

3 Os melhores resultados foram obtidos para $n=3,0 \mathrm{e}|g r|=0,10 \mathrm{~m}$.
}

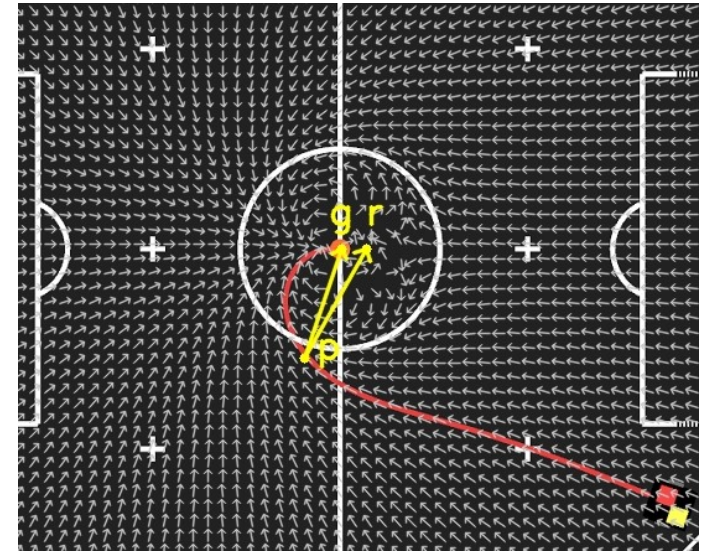

Figura 2. Campo vetorial atrativo (CVA). O caminho planejado é mostrado em vermelho, sendo obtido pela integração do campo vetorial a partir da posição inicial do robô. O ponto $g$ representa o objetivo, o ponto $r$ define a orientação de chegada ao objetivo e o ponto $p$ é uma posição qualquer no campo.

o modo mais simples é definir esse campo vetorial por um conjunto de vetores unitários obtidos pela seguinte função angular $\phi_{r}(p)$ :

em que $o$ é um obstáculo puntual.

$$
\phi_{r}(p)=-\angle \overrightarrow{p o}=\measuredangle \overrightarrow{o p}
$$

Porém, como proposto por Lim et al. (2008), é interessante possibilitar que o robô quando já está próximo do obstáculo e/ou está aproximando dele com maior velocidade possa reagir com certa antecipação. Para tal uma posição virtual do obstáculo, $o_{\text {virtual }}$, é obtida, levando em consideração a distância entre o obstáculo e o robô, $d$, e da relação entre suas velocidades, dada por:

$$
o_{\text {virtual }}= \begin{cases}o+\frac{d}{\left|\overrightarrow{p_{v}}\right|} \overrightarrow{p_{v}}, & \text { se } d<\left|\overrightarrow{p_{v}}\right| \\ o+\overrightarrow{p_{v}}, & \text { se } d \geq\left|\overrightarrow{p_{v}}\right|\end{cases}
$$

em que $\overrightarrow{p_{v}}$ é um vetor dado por:

$$
\overrightarrow{p_{v}}=K_{0}\left(v_{o b s}-v_{\text {robo }}\right)
$$

deslocando o centro de repulsão, mostrado na Figura 3 e ajustado pela constante $K_{0}$. Assim, o campo vetorial de desvio pode ser obtido pelos vetores unitários definidos pela função angular $\phi_{C V D}(p)$ :

$$
\phi_{C V D}(p)=\longdiv { \overline { o } _ { \text { virtual } } p }
$$

\subsection{Composição dos campos vetoriais}

Dado os dois campos vetoriais obtidos nas subseções anteriores, é necessário encontrar uma forma de combiná-los, gerando um campo vetorial que permita chegar ao alvo com uma determinada orientação e desviar dos obstáculos em seu percurso.

Observe que quando o robô está próximo ao obstáculo, $d \leq$ $d_{\min }$, a melhor solução é repeli-lo daquela posição. Porém, quando o robô está mais afastado do obstáculo, $d>d_{\min }$, é interessante adotar uma solução que permita afastar do obstáculo e, ao mesmo tempo, ir em direção ao alvo, ou seja, contornando o 


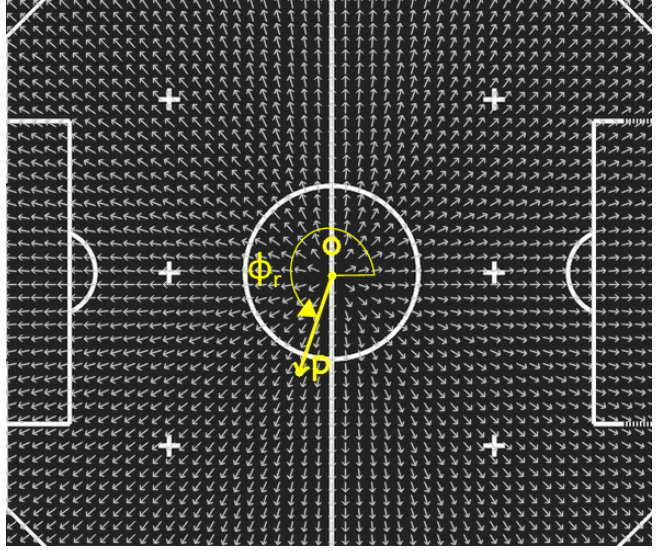

Figura 3. Campo vetorial puramente repulsivo centrado no ponto onde há um obstáculo, identificado pela letra $o$.

obstáculo se ele existir. Assim, pode-se definir o campo vetorial final como:

$$
\phi(p)=\left\{\begin{array}{l}
\phi_{C V D}(p), \text { se } d \leq d_{\min } \\
(1-k) \phi_{C V A}(p)+k \phi_{C V D}(p), \text { se } d>d_{\text {min }}
\end{array}\right.
$$

em que $k \in[0,1]$ é uma função que pode ser vista como uma porcentagem de ponderação entre o desvio e o direcionamento ao alvo. Além disso, essa função deve variar de acordo com a distância entre o robô e cada obstáculo, de tal maneira que quando não há obstáculos ou os obstáculos estão muito longe do robô, o campo vetorial composto deve ser diretamente aquele que leva o robô ao alvo $(k=0)$. Note, também, que fazendo $k=1$ tem-se a situação de $d \leq d_{\min }$.

Uma possibilidade para essa função é proposta no trabalho de Lim et al. (2008), sendo definida como uma função gaussiana com média igual a $d_{\min }$ e desvio padrão $\sigma$, ajustado conforme a suavidade desejada ${ }^{4}$ :

$$
k(d, \sigma)=e^{-\frac{\left(d-d_{\min }\right)^{2}}{2 \sigma^{2}}}
$$

Na Figura 4 é possível visualizar o caminho planejado no início do movimento do robô, integrando o campo vetorial obtido pela composição dos campos vetoriais. Observe que longe dos obstáculos o campo direciona diretamente para o alvo e próximo aos obstáculos o campo permite que o robô os contorne.

Por fim, a cada instante de tempo um novo $\phi$ é calculado para a posição atual do robô, definindo as velocidades em cada eixo a serem seguidas pelo robô:

$$
\left[\begin{array}{l}
\dot{x} \\
\dot{y}
\end{array}\right]=\left[\begin{array}{l}
v_{\text {Max }} \cdot \cos (\phi) \\
v_{\text {Max }} \cdot \sin (\phi)
\end{array}\right]
$$

em que $v_{\text {Max }}$ é a velocidade máxima que o robô consegue alcançar.

\section{SEGUIMENTO DO CAMPO VETORIAL}

O sistema de tração utilizado no robô é o diferencial, em que a variação da velocidade relativa das duas rodas é o que permite a alteração da sua velocidade linear $(v)$ e da sua velocidade angular $(\omega)$ e, consequentemente, da sua posição $(p)$ e da

\footnotetext{
4 A distância miníma escolhida na qual o robô irá sofrer efeitos puramente repulsivos foi definida para $d_{\min }=0,07 \mathrm{~m}, \delta=4,5$.
}

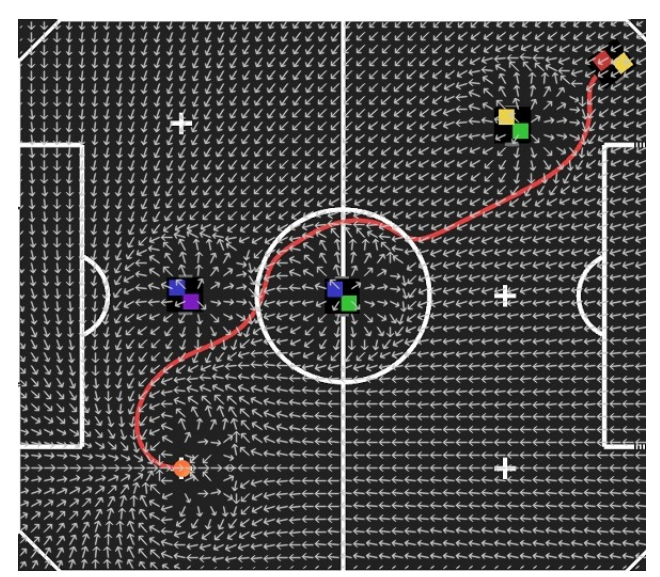

Figura 4. Campo vetorial final, composto pelo campos vetoriais de desvio e de atração ao alvo e indicado pelas setas. Já o caminho inicial obtido pela integração desse campo vetorial é mostrado em vermelho e a orientação final desejada foi definida como sendo igual a $\pi$.

sua orientação $(\theta)$. Assim, a Equação 9 apresenta o modelo cinemático do robô diferencial jogador de futebol:

$$
\dot{q}=\left[\begin{array}{c}
\dot{x}_{c} \\
\dot{y}_{c} \\
\dot{\theta}
\end{array}\right]=\left[\begin{array}{cc}
\cos (\theta) & 0 \\
\sin (\theta) & 0 \\
0 & 1
\end{array}\right] \cdot\left[\begin{array}{c}
v \\
\omega
\end{array}\right]
$$

em que $q=(p, \theta)$ é a configuração do robô, $\dot{x}_{c}$ e $\dot{y}_{c}$, respectivamente, a velocidade do centro do robô em relação ao eixo $x \mathrm{e}$ ao eixo $y$ no referencial adotado.

Diante desse modelo não-linear e do fato que o planejador, como descrito na Seção 4, fornece a cada instante de tempo um vetor de velocidades nos eixos $x$ e $y$, escolheu-se para seguir esse campo vetorial de velocidades a lei de controle descrita pela Equação 10:

$$
u_{1}(t)=\left[\begin{array}{c}
v \\
\omega
\end{array}\right]=\left[\begin{array}{cc}
\cos \theta & \sin \theta \\
-\frac{\sin \theta}{d_{r}} & \frac{\cos \theta}{d_{r}}
\end{array}\right] \cdot\left[\begin{array}{c}
\dot{x} \\
\dot{y}
\end{array}\right]
$$

em que $d_{r}$ é a distância do centro do robô até um ponto a frente do mesmo, de tal forma que quanto menor for o valor de $d_{r}$ maior serão as variações sentidas pelo robô quando acontecerem pequenas variações no ponto que se está seguindo e quanto maior for o valor de $d_{r}$ mais distante o centro do robô estará do caminho previsto. Esse tipo de controle é conhecido como controle via linearização por realimentação de entrada-saída (Feedback Linearization) e mais detalhes podem ser encontrados em (Chernousko, 2008).

Por fim, a partir da Equação 10, é possível determinar o sinal de controle $u(t)$, constituído pelas velocidades angulares de cada roda a serem executadas pelo robô:

$$
u(t)=\left[\begin{array}{c}
\omega_{r_{\text {direita }}} \\
\omega_{r_{\text {esquerda }}}
\end{array}\right]=\left[\begin{array}{cc}
1 / r & L / r \\
1 / r & -L / r
\end{array}\right] \cdot\left[\begin{array}{c}
v \\
\omega
\end{array}\right]
$$

em que $L$ é a largura do robô (distância entre os centro das rodas) e $r$ é o raio das rodas.

\section{EXPERIMENTOS E RESULTADOS}

Por meio de simulações, avaliou-se no VSS-Viewer o funcionamento geral do planejamento de caminho, utilizando o campo vetorial apresentado na Seção 4, e do controle para o seguimento do campo vetorial descrito na Seção 5. Com base 


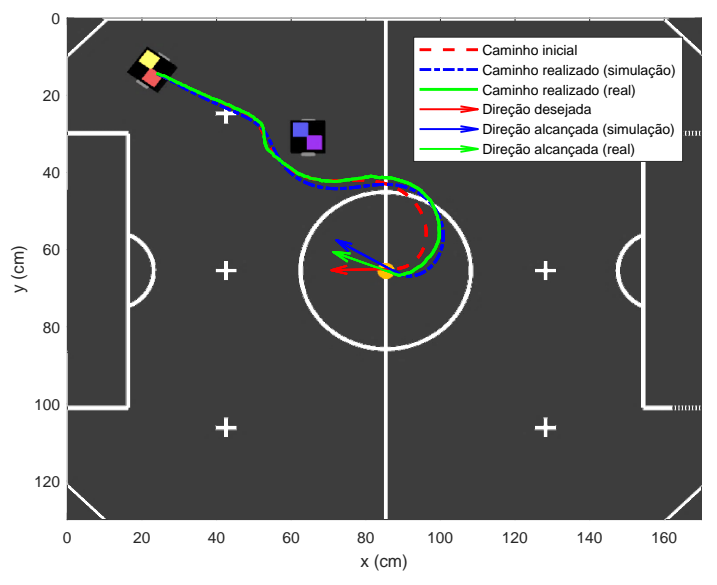

Figura 5. Comparação entre o caminho inicial planejado e o realizado pelo robô na simulação e no experimento real.

no sucesso desses resultados, foram realizados os experimentos com os robôs reais no ambiente de uma partida de futebol de robôs.

Dessa forma, na Figura 5 é apresentada uma comparação entre o caminho inicial planejado e o realizado pelo robô na simulação e no experimento real. Repare que o caminho realizado no experimento real é muito próximo daquele da simulação e que o robô consegue desviar do obstáculo.Além disso, o robô chega até a bola com uma orientação próxima àquela desejada, com um erro médio de 0,35 rad.

Com o intuito de verificar o funcionamento do planejamento proposto, tomou-se duas situações ordinárias de um jogo de futebol: (i) situação de ataque, em que o robô atacante deve alcançar a bola e ir em direção ao gol adversário e (ii) situação de defesa, em que o robô defensor deve alcançar a bola para proteger o próprio gol. Em ambas as situações dois robôs foram colocados para dificultar o percurso do robô, sendo necessário que o robô atacante/defensor os desviasse ${ }^{5}$. O resultado desses experimentos podem ser vistos, respectivamente, na Figura 6 e na Figura 7. Note que o caminho inicial é apenas apresentado para se ter uma referência do caminho a ser executado pelo robô, pois a cada instante o robô re-calcula o campo vetorial correspondente a sua posição, dada a posição dos obstáculos e do alvo. Pode-se perceber ainda que em alguns momentos o caminho realizado pelo robô tem mudanças bruscas que podem ser devidas ao fato que o campo vetorial proposto em alguns pontos, principalmente, próximo aos obstáculos, é pouco suave ou, até mesmo, devidas às limitações físicas impostas pelo robô e pelo ambiente. Um vídeo desse experimento pode ser visto em: https://youtu.be/kIyQ7nDE2lg.

Pela Equação 10, percebe-se que um parâmetro importante do controle implementado é a distância escolhida de controle, $d_{r}$. Para verificar a sua influência no seguimento do campo vetorial proposto, foi realizado outro experimento com um robô real, em uma região livre de obstáculos, como mostrado pela Figura 8. Observe que quanto maior é $d_{r}$ mais suave é o caminho realizado e, em contrapartida, esse caminho é mais distante do caminho inicial, já que a curva realizada será

\footnotetext{
5 Essa escolha foi feita motivada pela ocorrência mais frequente desse tipo de situação em que apenas dois robôs bloqueiam o atacante/defensor de alcançar a bola.
}

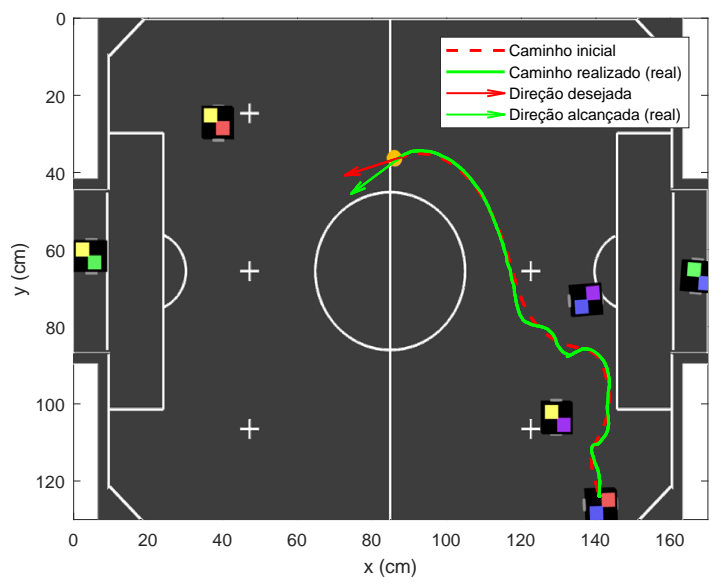

Figura 6. Caminho realizado pelo robô para alcançar a bola com uma orientação direcionada para gol, desviando dos obstáculos no percurso (situação de ataque).

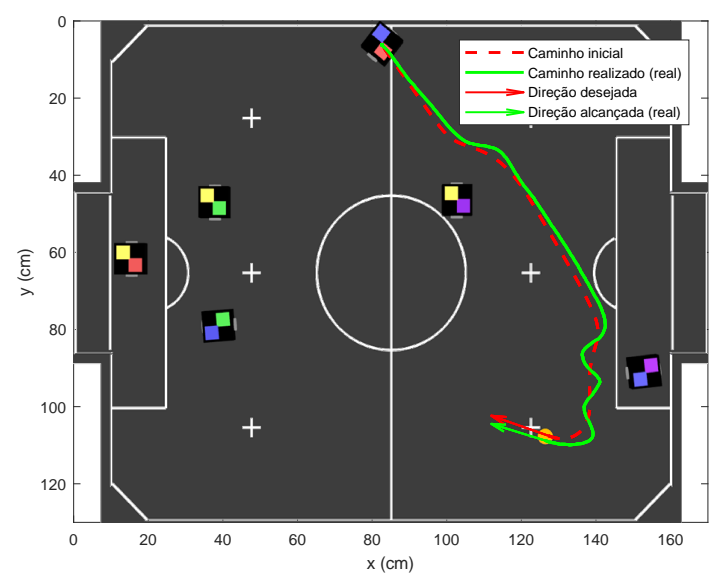

Figura 7. Caminho realizado pelo robô para alcançar a bola com uma orientação direcionada para gol, desviando dos obstáculos no percurso (situação de defesa).

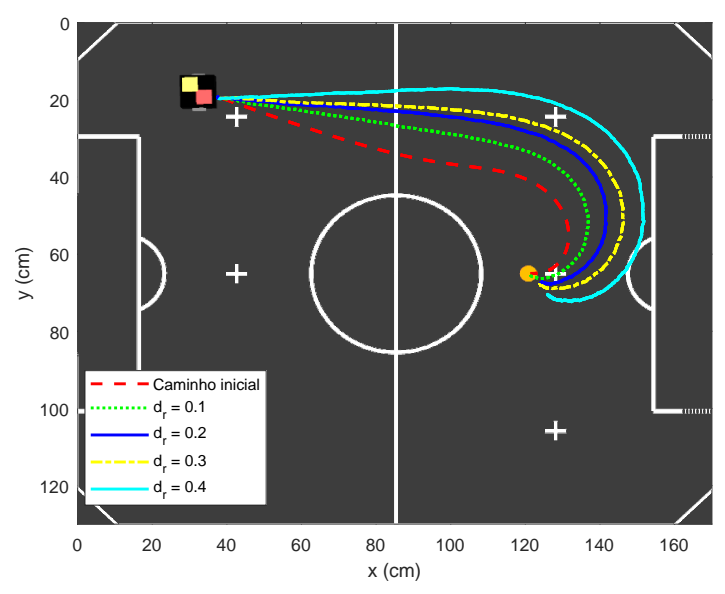

Figura 8. Variação do caminho realizado pelo robô, ao variar o parâmetro de controle $d_{r}$ (em metros).

maior. O valor de $d_{r}$ escolhido nos experimentos foi de $0,10 \mathrm{~m}$, próximo a extremidade do robô.

Ainda do ponto de vista do controle, implementou-se o controle por modos deslizantes proposto por (Kim et al., 2001) 


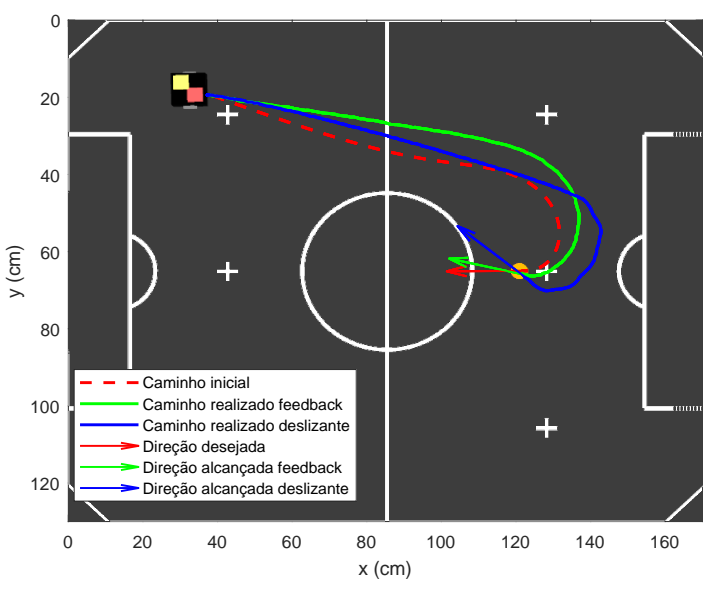

Figura 9. Comparação entre o caminho realizado pelo robô real, utilizando para o seguimento do caminho o controle feedback linearization e o controle por modos deslizantes.

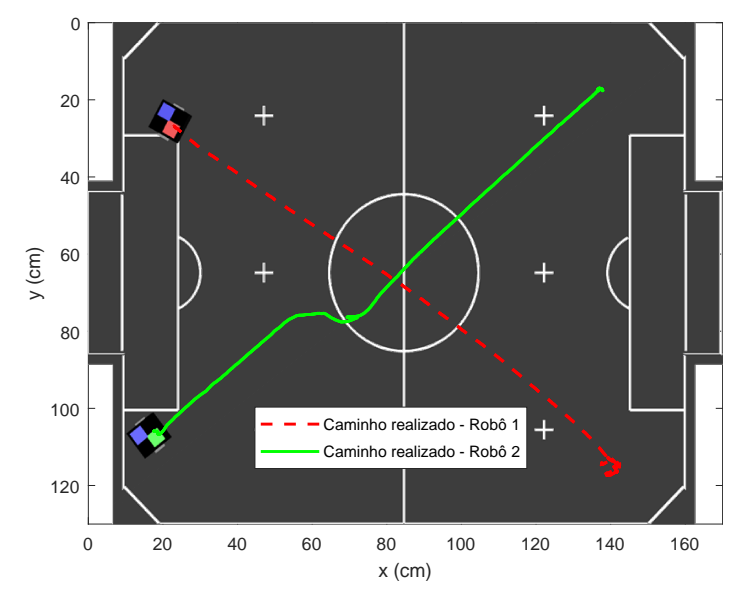

Figura 10. Desvio dinâmico utilizando o campo vetorial proposto.

e ajustado para o melhor caso testado, para comparar com o utilizado neste trabalho. Nota-se pela Figura 9 que o controle feedback linearization obteve um resultado melhor, mantendo o robô mais próximo do caminho inicial e chegando ao alvo com um erro de orientação muito menor do que utilizando o controle por modos deslizantes.

Por fim, foi realizado um experimento considerando um obstáculo em movimento que cruza com o caminho previsto de outro robô, como pode ser visto na Figura 10 e com mais detalhes em: https://youtu.be/pyVjGjFc4Eg. Esses resultados confirmam, também, o sucesso do desvio dinâmico do planejamento proposto.

\section{CONCLUSÃO E TRABALHOS FUTUROS}

Este trabalho apresentou o desenvolvimento de robôs diferenciais jogadores de futebol para a categoria IEEE Very Small Size Soccer. De maneira geral foi descrito cada subsistema necessário para possibilitar a navegação autônoma do robô no campo. Como uma das contribuições, foi apresentada e validada uma combinação de campos vetoriais que possibilitam o planejamento de caminho do robô futebolista.
Os resultados e comparações realizadas com os robôs reais apontam que a técnica adotada permite um ganho na eficiência do robô durante o jogo, já que o mesmo além de chegar à bola com a orientação definida pela estratégia geral de jogo, por exemplo, com a orientação apontada para o gol, permite que o robô desvie dos possíveis obstáculos em seu caminho, evitando o tempo gasto nas colisões e de uma possível estratégia de marcação do adversário. Também foi possível por meio dos experimentos validar o desvio de obstáculos dinâmicos, garantindo nessas situações um deslocamento livre de colisões.

Trabalhos futuros visam melhorar o controle do robô, acrescentando um controle embarcado, de forma que o robô execute o mais próximo possível as velocidades fornecidas pelo planejador. Além de implementar novos campos vetoriais que possibilitem caminhos mais suaves, reduzindo o tempo gasto para a correção de possíveis movimentos bruscos.

\section{REFERÊNCIAS}

Chernousko, F. (2008). Control of Nonlinear Dynamical Systems. Springer-Verlag, Berlin, 1 edition.

Freitas, E.J.R., Passos, H.A., and Pereira, G.A.S. (2017). Desvio de obstáculos por robôs semiautônomos usando planejamento de caminhos. XIII Simpósio Brasileiro de Automação Inteligente, 1043-1048.

Hassan, K.M. (2014). Design an intelligent proportionalderivative (pd) feedback linearization control for nonholonomic-wheeled mobile robot. Journal of Intelligent; Fuzzy Systems, 26(4), 1833-1843. doi:10.3233/IFS-130863.

He, S. (2005). Feedback control design of differential-drive wheeled mobile robots. In ICAR05. Proceedings., 12th International Conference on Advanced Robotics, 2005. IEEE. doi:10.1109/icar.2005.1507403.

Junkai, R., Chenggang, X., Junhao, X., Kaihong, H., and Huimin, L. (2016). A control system for active ball handling in the robocup middle size league. In 2016 Chinese Control and Decision Conference (CCDC). IEEE.

Kim, J.H., Kim, D.H., Kim, Y.J., and Seow, K.T. (2010). Soccer Robotics (Springer Tracts in Advanced Robotics). Springer.

Kim, Y.J., Kim, J.H., and Kwon, D.S. (2001). Evolutionary programming-based univector field navigation method for past mobile robots. IEEE Transactions on Systems, Man, and Cybernetics, Part B (Cybernetics), 31(3), 450-458.

Li, T.H., Chen, C.Y., Lee, S., and Guo, Y.Z. (2003). Design and implementation of fuzzy ring univector field for robot soccer game. In Proceedings 2003 IEEE International Symposium on Computational Intelligence in Robotics and Automation. Computational Intelligence in Robotics and Automation for the New Millennium (Cat. No. 03EX694), volume 1, 85-90. IEEE.

Lim, Y., Choi, S.H., Kim, J.H., and Kim, D.H. (2008). Evolutionary univector field-based navigation with collision avoidance for mobile robot. In Proc. 17th World Congress The International Federation of Automatic Control, Seoul, Korea (July 2008).

Park, K.H., Kim, Y.J., and Kim, J.H. (2001). Modified univector field navigation and modular q-learning for soccer robots. In Proceedings of the 32nd ISR (International Symposium on Robotics), volume 19, 21.

Vien, N.A., Viet, N.H., Park, H., Lee, S., and Chung, T. (2007). Q-learning based univector field navigation method for mobile robots. In Advances and Innovations in Systems, Computing Sciences and Software Engineering, 463-468. Springer. 